\title{
Estrutura da população e aspectos reprodutivos do peixe-rei Atherinella brasiliensis (Quoy \& Gaimard) (Atheriniformes, Atherinopsidae) em áreas rasas do complexo estuarino de Paranaguá, Paraná, Brasil
}

\author{
Luís F. Fávaro ${ }^{1}$; Elton C. de Oliveira ${ }^{1} \&$ Nelsy F. Verani ${ }^{2}$ \\ 1 Laboratório de Reprodução e Comunidade de Peixes, Departamento de Biologia Celular, Universidade Federal do Paraná. \\ Caixa Postal 19031,81531-980 Curitiba, Paraná, Brasil.E-mail: lufavaro@ufpr.br; elton_oliveirabio@yahoo.com.br \\ 2 Departamento de Hidrobiologia, Centro de Ciências Biológicas e da Saúde, Universidade Federal de São Carlos. \\ Rodovia Washington Luís, km 235, $13565-905$ São Carlos, São Paulo, Brasil. E-mail: dnfv@power.ufscar.br
}

\begin{abstract}
Structure of the population and reproductive aspects of silverside Atherinella brasiliensis (Quoy \& Gaimard) (Atheriniformes, Atherinopsidae) in shallow areas of the estuarine complex Paranaguá, Paraná, Brazil. The population structure and reproductive aspects of Atherinella brasiliensis (Quoy \& Gaimard, 1825) had been analyzed monthly from May 2000 to April 2001 in eight points of shallow areas of the estuarine complex Paranaguá. The environment analyzes of the parameters $\mathrm{pH}$, dissolved oxygen, transparency and salinity of the water suggest the existence of an increasing environment gradient in the direction North-South highway of the estuary. The temperature of the water evidenced the seasonal variation in the studied system, revealing related with the reproductive process. The peak of reproductive activity was determined for females and male, respectively, in the months of October and September, period where if it verified the ratio of two females for a male. The predominance of young individuals in the estuary occurred in the end of the spring and during the summer, after the reproductive period. The seasonal analyses of length and mass suggest the existence of a cyclical standard of growth to the long of the year, which reflects the reproductive period and the entrance of young individuals in the environment. Thus, it is concluded that $A$. brasiliensis is a resident estuarine species, revealing widely distributed in the estuarine shallow areas, being able to be considered key in evaluations of environmental impact and/or action of handling plans and conservation in estuarine environments.

KEY WORDS. Corporal mass structure; reproductive cycle; structure in length.
\end{abstract}

RESUMO. A estrutura populacional e aspectos reprodutivos de Atherinella brasiliensis (Quoy \& Gaimard, 1825) foram analisados mensalmente de maio de 2000 a abril de 2001, em oito pontos de ambientes rasos do eixo norte-sul do complexo estuarino de Paranaguá. As análises ambientais das variáveis $\mathrm{pH}$, oxigênio dissolvido, transparência e salinidade da água sugerem a existência de um gradiente ambiental crescente no sentido nortesul do estuário. A temperatura da água evidenciou a variação temporal no sistema estudado, mostrando-se intimamente relacionado com o processo reprodutivo. O pico de atividade reprodutiva foi determinado para fêmeas e machos, respectivamente, nos meses de outubro e setembro, período em que se verificou a proporção de duas fêmeas para um macho. O predomínio de indivíduos jovens no estuário ocorreu no final da primavera e durante o verão, após o período reprodutivo. As análises sazonais de comprimento e de massa corporal sugerem a existência de um padrão cíclico de crescimento ao longo do ano, o qual reflete o período reprodutivo e o recrutamento. Assim, conclui-se que $A$. brasiliensis é uma espécie estuarina residente, mostrando-se amplamente distribuída nas áreas rasas estuarinas, podendo ser considerada chave em avaliações de impacto ambiental e/ ou em ações de planos de manejo e conservação em ambientes estuarinos.

PALAVRAS-CHAVE. Ciclo reprodutivo; estrutura em comprimento; estrutura em massa corporal.

O peixe-rei, Atherinella brasiliensis (Quoy \& Gaimard, 1825), é encontrado em águas salobras e se distribui da Venezuela ao Rio Grande do Sul (Figueiredo \& Menezes 2000). É considerada uma espécie tipicamente estuarina (BARBIERE et al.
1991, Pessanha \& Araújo 2001), sendo descrita em vários trabalhos como uma das mais abundantes em áreas rasas estuarinas do sudeste do Brasil (BARbiere et al. 1991, ANDreata et al. 1997, Pessanha \& Araújo 2001).

Revista Brasileira de Zoologia 24 (4): 1150-1156, dezembro 2007 
Por ser a espécie considerada abundante e constante no complexo estuarino de Paranaguá (Godefroid et al. 1997, SANTOS et al. 2002), este trabalho objetiva analisar a estrutura da população e a biologia reprodutiva de $A$. brasiliensis em diferentes pontos amostrais de áreas rasas no complexo estuarino de Paranaguá. Esses objetivos justificam-se pela abundância e ampla distribuição da referida espécie neste ambiente, pela escassez de trabalhos de estrutura populacional no estuário em questão e para complementar o estudo de reprodução realizado por FÁvARO et al. (2003), o qual foi realizado em um único ponto amostral na baía de Paranaguá. Assim, espera-se contribuir para a compreensão do funcionamento dos ecossistemas estuarinos e, se necessário, subsidiar políticas de conservação e planos de manejo da espécie.

\section{MATERIAL E MÉTODOS}

O complexo estuarino de Paranaguá abrange um grande corpo aquoso de forma irregular que se projeta continente adentro na porção norte da planície litorânea do Paraná $\left(25^{\circ} 15^{\prime} \mathrm{S}\right.$ e $48^{\circ} 45^{\prime} \mathrm{W} \& 25^{\circ} 35^{\prime} \mathrm{S}$ e $48^{\circ} 10^{\prime} \mathrm{W}$ ). Este sistema estuarino encontrase dividido em dois eixos principais de orientação, o eixo lesteoeste, formado pelas baías de Antonina e de Paranaguá, e o eixo norte-sul, formado pelas baías das Laranjeiras e de Guaraqueçaba (Spvs 1992).

Segundo a classificação de Köppen, o clima da região é o Cfa, com verão quente e sem estação seca definida. A temperatura do ar do mês mais quente apresenta uma média acima de $22^{\circ} \mathrm{C}$ e a do mês mais frio uma média abaixo de $18^{\circ} \mathrm{C}$ (MAACK 1981). A região apresenta umidade média de $85 \%$ (Bigarella et al. 1978) e uma média anual de precipitação de $2000 \mathrm{~mm}$ (SUDERHSA 1998).

Coletas mensais foram realizadas com rede de arrasto manual (30,0 m de comprimento x 2,0 m de altura, 2,0 m de boca, com malhagem de $0,5 \mathrm{~cm}$ entre os nós adjacentes) no período de maio de 2000 a abril de 2001. Foram efetuados dois arrastos consecutivos de 50 metros em cada um dos oito pontos amostrais (P1, P2, P3, P4, P5, P6, P7 e P8), localizados em áreas rasas, no eixo norte-sul do complexo estuarino de Paranaguá (Fig. 1). Através da distribuição dos pontos amostrais, estabeleceu-se que os pontos P1, P2, P3 e P4 localizam-se ao norte, os pontos P5 e P6 ao centro e os pontos P7 e P8 ao sul do estuário.

Foram capturados 16147 indivíduos, dos quais 3691, amostrados aleatoriamente, tiveram registrados os dados de comprimento total $(\mathrm{cm})$ e peso total $(\mathrm{g})$. O excedente, ou seja, 12456 indivíduos foram contados e o peso total registrado.

Dos 3691 indivíduos que tiveram os dados morfométricos tomados, uma sub amostragem aleatória destinou 1845 espécimes ao estudo reprodutivo (611 fêmeas, 439 machos e 795 sem sexo identificado). Após secção ventral, estes exemplares tiveram as gônadas analisadas macroscopicamente quanto ao sexo e ao desenvolvimento gonadal. Ovários e testículos foram retirados, pesados (g) e analisados microscopicamente para a confirmação e correção das análises macroscópicas quando necessário.

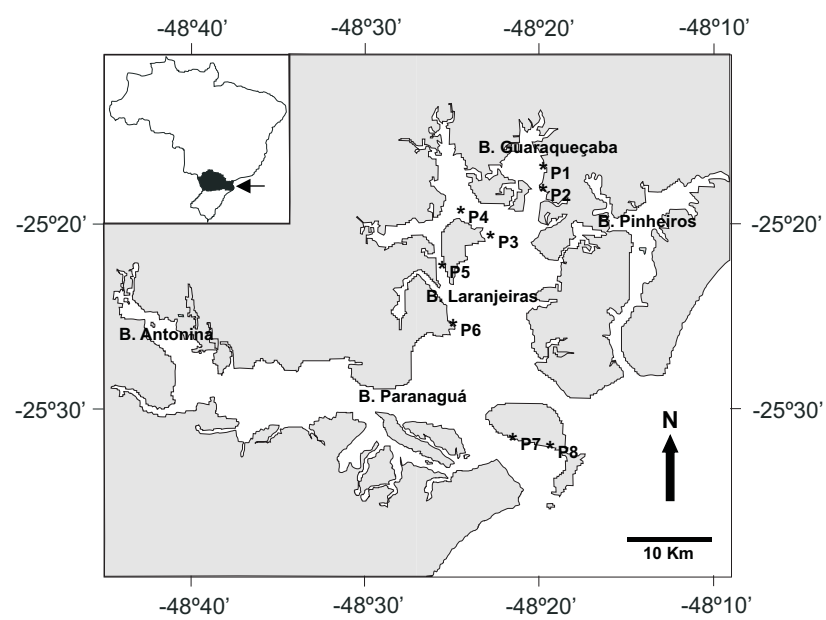

Figura 1. Complexo estuarino de Paranaguá, litoral norte do Paraná, com a localização dos pontos amostrais.

Simultaneamente à captura de material biológico foram coletados dados físicos e químicos da água: salinidade (utilizando-se refratômetro), transparência (com Disco de Secchi), $\mathrm{O}_{2}$ dissolvido (método de Winkler, de acordo com Grasshoff et al. (1983)), pH (com potenciômetro) e temperatura (com termômetro de mercúrio), os quais a partir de uma matriz foram analisados através de Análise Discriminante.

Os 3691 exemplares que tiveram os dados morfométricos tomados foram distribuídos em classes de comprimento, determinadas de acordo com o Postulado de Sturges (SoKAL \& RoHLF 1981).

A estrutura em comprimento foi avaliada através das freqüências absolutas e percentuais dos indivíduos por classe de comprimento, sendo analisadas: 1) sazonalmente para o conjunto dos pontos amostrais; 2) para fêmeas (611) e machos (439), separadamente, no conjunto dos pontos amostrados.

A estrutura em massa corporal foi analisada sazonalmente para o conjunto dos pontos amostrados, utilizando os 16147 indivíduos obtidos. Cada ponto amostral teve a massa corporal total registrada, a partir da soma das massas corporais de cada indivíduo. Foram determinados os valores sazonais da massa corporal média em cada ponto amostral através da relação entre a massa corporal total e o número de indivíduos em cada estação.

O ciclo reprodutivo foi verificado, separadamente para fêmeas e machos, com base nos valores do Índice GônadoSomático (IGS), expresso pela fórmula: $\mathrm{IGS}=(\mathrm{Pg} / \mathrm{Pt})^{\star} 100$, onde $\mathrm{Pg}$ = peso da gônada e Pt = peso total do indivíduo.

A partir dos valores individuais do IGS foi calculado o IGS médio mensal para a confecção da curva de maturação de ambos os sexos. Esta análise deu-se para o conjunto de pontos amostrais e para cada ponto em separado.

A proporção sexual foi determinada para os 1050 indivíduos que tiveram o sexo identificado no período de estudo, considerando o conjunto dos pontos (sazonal) e as classes de 
comprimento. As análises foram testadas pelo método do $\chi^{2}$, com grau de liberdade 1 e 0,05 de significância $(\alpha=0,05)$, de acordo com VAzzoler (1996).

Para a determinação da proporção de jovens e adultos foram utilizados 1845 exemplares, sendo considerados indivíduos jovens aqueles que apresentaram gônadas no estádio imaturo (A) e adultos os exemplares que apresentaram gônadas nos estádios: maturação (B), maduro (C) ou desovado/esvaziado (D). Foi realizada a distribuição mensal de indivíduos jovens e adultos de $A$. brasiliensis para pontos agrupados.

A análise foi efetuada considerando o comprimento de primeira maturação de fêmeas e machos de A. brasiliensis determinado por FÁvARo et al. (2003) e pela análise microscópica das gônadas, realizada para a confirmação dos estádios de maturação de indivíduos de comprimentos reduzidos.

Neste trabalho, as estações do ano foram definidas como sendo: primavera, de setembro a novembro; verão, de dezembro a fevereiro; outono, de março a maio e inverno, de junho a agosto.

\section{RESULTADOS}

A partir da análise discriminante realizada com os fatores físicos e químicos da água observou-se a maior influência do $\mathrm{pH}$, além de evidenciar a importância da salinidade, do oxigênio dissolvido e da transparência da água. A temperatura da água caracterizou a variação temporal, indicando o efeito da sazonalidade (Fig. 2). Constatou-se a existência de um gradiente crescente dos fatores físicos e químicos da água, no sentido norte-sul do complexo estuarino de Paranaguá, ou seja, maiores valores de $\mathrm{pH}$, de temperatura, de salinidade, de oxigênio dissolvido e de transparência foram registrados nos pontos localizados ao sul (Fig. 3).

A análise sazonal da distribuição espacial e temporal (Tab. I) registrou o maior número de indivíduos capturados no verão, seguido pelo inverno, outono e primavera. Os pontos de maior e menor captura de A. brasiliensis foram o P6 e P8, respectivamente. No ponto $\mathrm{P} 6$ constatou-se as maiores capturas nas estações do inverno, primavera e outono com 61\%, 33\% e $41 \%$ do total capturado, respectivamente.

Dos exemplares capturados no complexo estuarino de Paranaguá, o menor e o maior tamanho foram, respectivamente, $1,9 \mathrm{~cm}$ e $15,8 \mathrm{~cm}$ de comprimento total, correspondendo a uma amplitude de $13,9 \mathrm{~cm}$.

No verão observou-se a maior freqüência de indivíduos
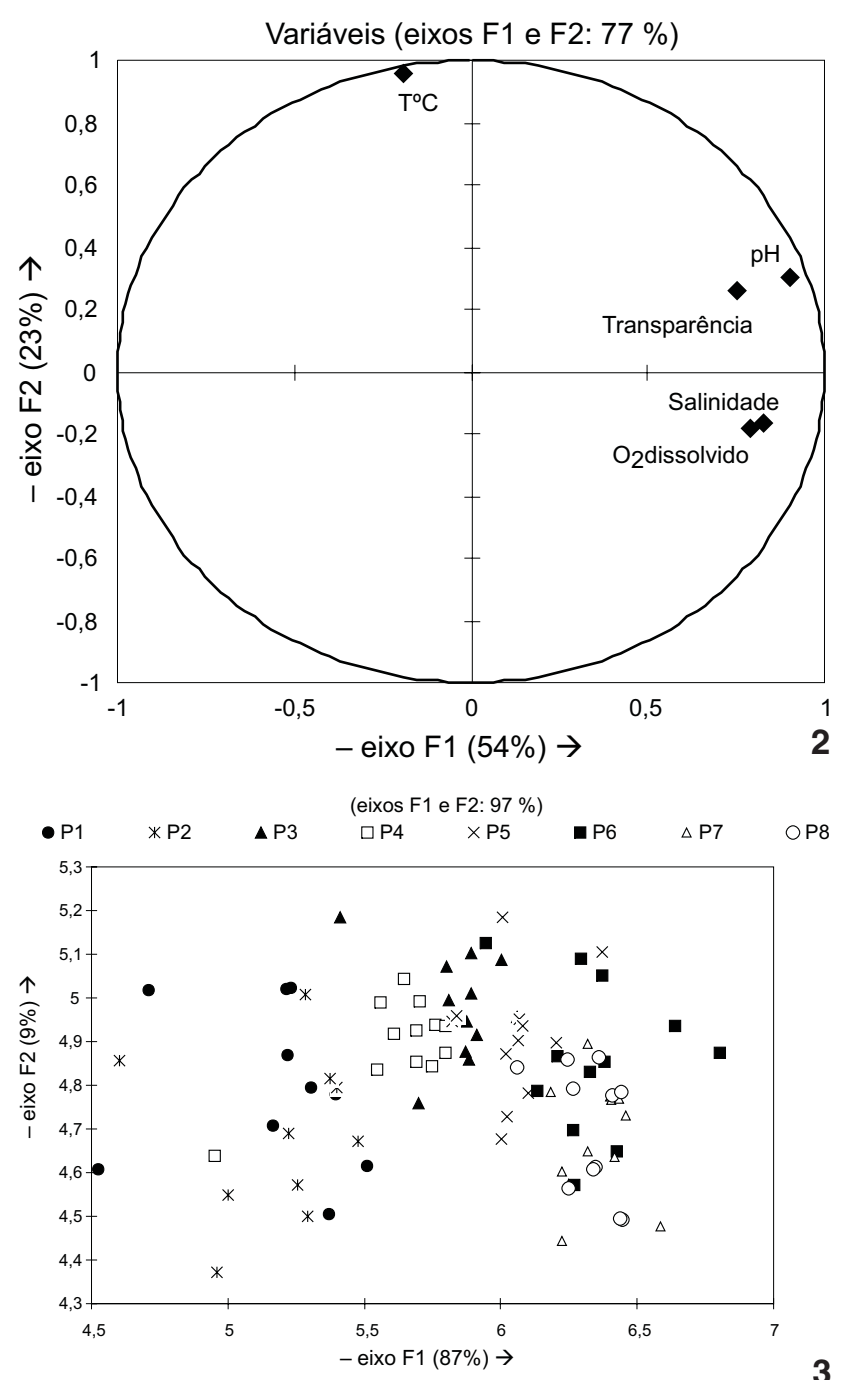

3

Figuras 2-3. Representação gráfica da Análise Discriminante na determinação do grau de importância dos fatores físicos e químicos da água (2) e do gradiente ambiental a partir dos dados físicos e químicos da água (3).

nas menores classes de comprimento, no outono nas classes intermediárias, no inverno nas classes intermediárias às maiores e na primavera nas maiores classes de comprimento (Fig. 4).

Tabela I. Número de indivíduos capturados sazonalmente em cada ponto amostral.

\begin{tabular}{lccccrrrrr}
\hline \multicolumn{1}{c}{ Mês } & P1 & P2 & P3 & P4 & P5 & P6 & P7 & P8 & Total mensal \\
\hline Inverno & 259 & 224 & 596 & 323 & 171 & 2729 & 107 & 35 & 4444 \\
Primavera & 478 & 312 & 115 & 144 & 82 & 611 & 65 & 31 & 1838 \\
Verão & 112 & 134 & 654 & 1387 & 843 & 1024 & 1725 & 131 & 6010 \\
Outono & 583 & 141 & 217 & 318 & 434 & 1594 & 439 & 129 & 3855 \\
\hline Total (ponto) & 1432 & 811 & 1582 & 2172 & 1530 & 5958 & 2336 & 326 & 16147 \\
\hline
\end{tabular}




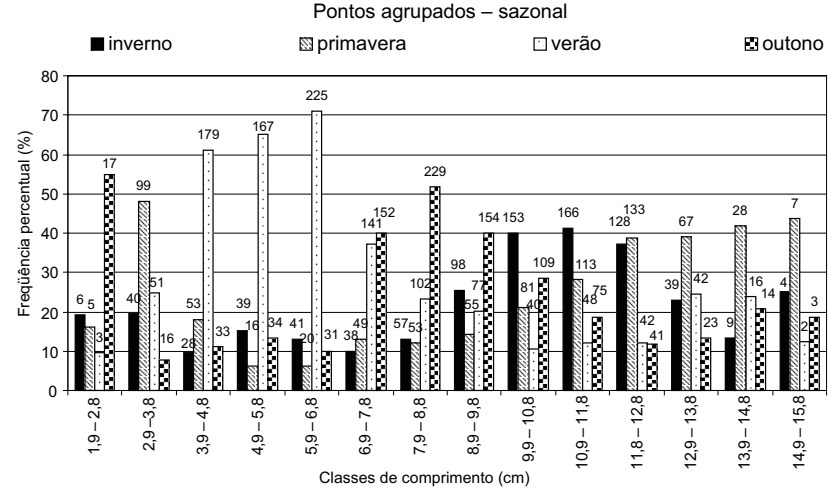

Figura 4. Distribuição sazonal dos indivíduos de $A$. brasiliensis em classes de comprimento para pontos agrupados. O valor acima da barra indica a freqüência absoluta sazonal nas classes de comprimento.

As fêmeas apresentaram predomínio na freqüência percentual de captura entre os comprimentos de 10,9 a 15,8 cm, enquanto os machos tiveram maior representatividade entre os tamanhos de 4,9 a 10,8 cm (Fig. 5). A análise gráfica permite verificar o maior tamanho das fêmeas.

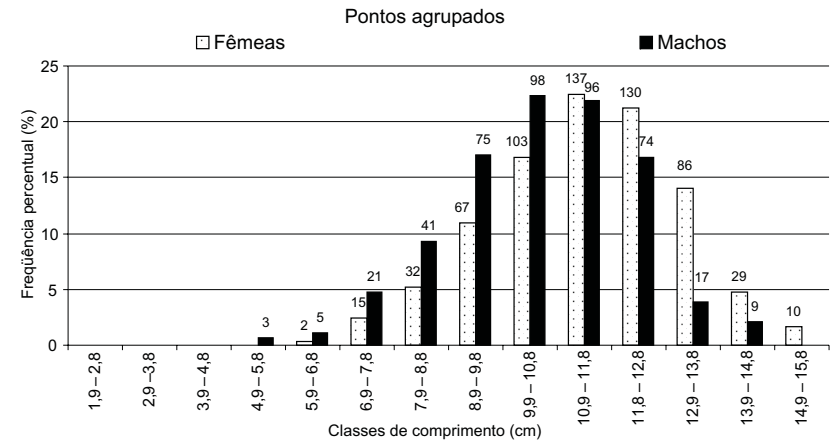

Figura 5. Distribuição de fêmeas e machos de $A$. brasiliensis em classes de comprimento para pontos agrupados. O valor acima da barra indica a freqüência absoluta nas classes de comprimento.

A massa corporal total registrada para A. brasiliensis foi de 79301,03g, sendo o inverno a estação de maior valor $(35139,19$ g), seguida do outono $(19368,98 \mathrm{~g})$, verão $(12638,67 \mathrm{~g})$ e primavera $(12159,19 \mathrm{~g})$.

Através da análise da massa corporal média sazonal constatou-se que, geralmente, os indivíduos de menor massa ocorreram no verão, os de maior massa na primavera e indivíduos com valores intermediários foram observados no outono e no inverno (Tab. II).

Através da análise da curva de maturação para pontos agrupados observou-se um aumento progressivo do desenvolvimento gonadal a partir de junho, alcançando em outubro o pico do IGS médio para as fêmeas e em setembro para os machos (Figs 6 e 7). Padrão reprodutivo semelhante foi constatado quando se analisaram os pontos isoladamente, indicando que as diferentes áreas estudadas foram utilizadas de maneira semelhante.
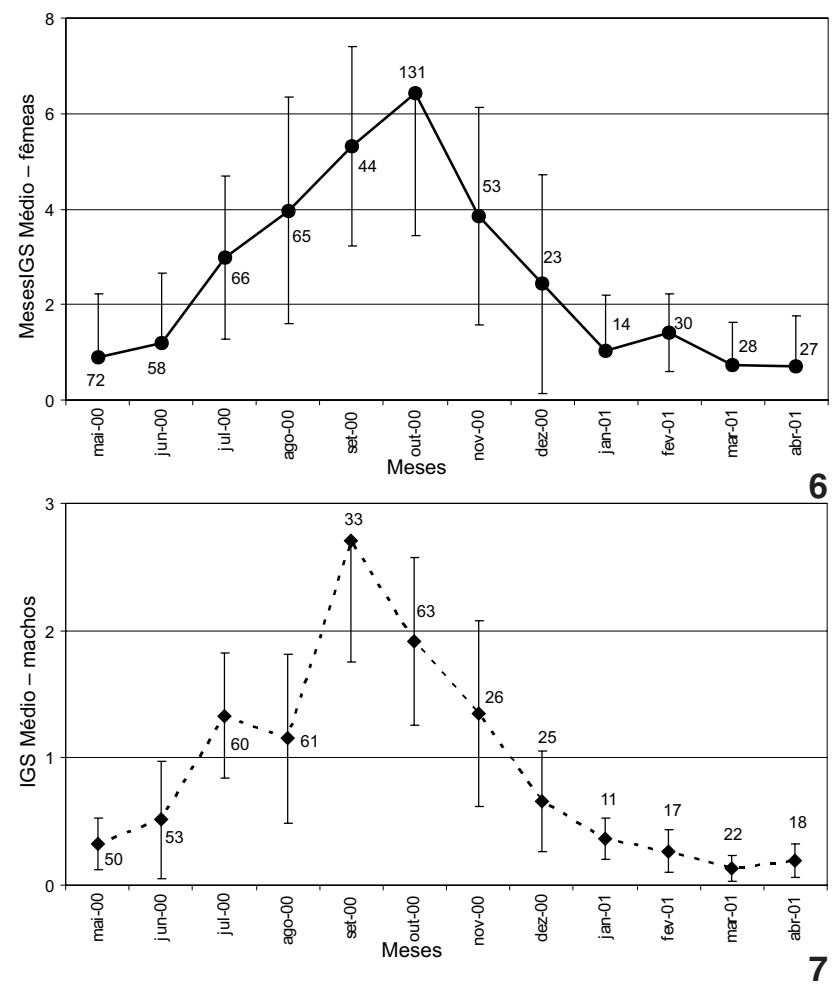

Figuras 6-7. Curva de maturação de fêmeas (6) e machos (7) (IGS Médio acompanhado do desvio padrão) de A. brasiliensis para pontos agrupados, maio de 2000 (mai-00) a abril de 2001 (abr01). Os números junto às curvas de maturação representam a freqüência absoluta mensal de indivíduos.

Sazonalmente, a proporção sexual para o conjunto de pontos registrou um predomínio de fêmeas em relação aos machos em todas as estações do ano, tendo na primavera, período de maior desenvolvimento gonadal, a relação aproximada de duas fêmeas para um macho (Tab. III).

A análise da proporção sexual por classe de comprimento (Tab. IV) revelou uma discreta superioridade numérica de machos entre os comprimentos de 4,9 a $9,8 \mathrm{~cm}$, enquanto as fêmeas apresentaram uma significativa predominância nos tamanhos que variaram de 9,9 a 15,8 cm.

A análise da proporção de jovens e adultos revelou um predomínio de adultos no sistema estudado, sendo registrados 1113 indivíduos adultos e 732 jovens. Este padrão não foi constatado durante todo o ciclo de coleta, uma vez que no verão ocorreu predomínio de jovens (Fig. 8). 
Tabela II. Massa corporal média sazonal com o desvio padrão (DP), massa corporal total por ponto amostral e massa corporal total por estação.

\begin{tabular}{|c|c|c|c|c|c|}
\hline \multirow{2}{*}{ Pontos } & Inverno & Primavera & Verão & Outono & \multirow{2}{*}{ Massa total (g) } \\
\hline & Média \pm (DP) & Média \pm (DP) & Média \pm (DP) & Média \pm (DP) & \\
\hline P1 & $4,94( \pm 3,16)$ & $5,50( \pm 4,41)$ & $4,13( \pm 5,51)$ & $10,15( \pm 3,09)$ & 10293,88 \\
\hline P2 & $6,24( \pm 3,74)$ & $6,48( \pm 4,59)$ & $3,04( \pm 3,14)$ & $6,72( \pm 3,35)$ & 4777,54 \\
\hline P3 & $7,69( \pm 4,03)$ & $9,59( \pm 7,04)$ & $1,76( \pm 3,19)$ & $5,29( \pm 3,59)$ & 7987,79 \\
\hline P4 & $8,30( \pm 3,31)$ & $6,86( \pm 5,33)$ & $2,37( \pm 1,77)$ & $3,20( \pm 2,24)$ & 7971,70 \\
\hline P5 & $7,31( \pm 2,87)$ & $6,99( \pm 4,80)$ & $1,74( \pm 2,40)$ & $4,32( \pm 2,02)$ & 5172,18 \\
\hline P6 & $8,14( \pm 2,72)$ & $5,80( \pm 5,22)$ & $3,12( \pm 2,38)$ & $3,82( \pm 1,62)$ & 35033,48 \\
\hline P7 & $12,39( \pm 4,32)$ & $12,99( \pm 7,63)$ & $1,06( \pm 1,16)$ & $4,17( \pm 1,77)$ & 5831,51 \\
\hline P8 & $11,34( \pm 3,81)$ & $14,48( \pm 5,12)$ & $6,42( \pm 21,14)$ & $4,23( \pm 1,15)$ & 2232,95 \\
\hline Massa total por estação & 35139,19 & 12154,19 & 12638,67 & 19368,98 & \\
\hline
\end{tabular}

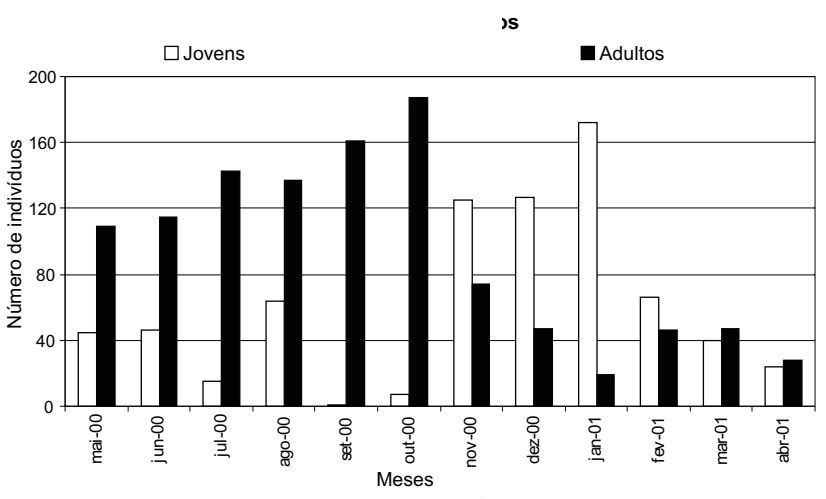

Figura 8. Distribuição mensal de indivíduos jovens e adultos de $A$. brasiliensis para pontos agrupados.

Tabela III. Proporção sexual sazonal de $A$. brasiliensis para pontos agrupados.

\begin{tabular}{lccc}
\hline \multicolumn{1}{c}{ Estação } & Fêmeas & Machos & $\chi^{2}$ \\
\hline Inverno & 189 & 174 & 0,62 \\
Primavera & 228 & 122 & $32,10^{*}$ \\
Verão & 67 & 53 & 1,63 \\
Outono & 127 & 90 & $6,31^{*}$ \\
\hline
\end{tabular}

* Valores que representam diferenças significativas quando testados pelo método do $\chi^{2}(\alpha<0,05)$.

\section{DISCUSSÃO}

As análises ambientais realizadas foram importantes para caracterizar cada local amostrado, buscando-se compreender a distribuição de $A$. brasiliensis nestes ambientes.

No presente trabalho o $\mathrm{pH}$ foi a variável aferida que mais influenciou na formação do gradiente ambiental em áreas rasas ao longo do eixo norte-sul do complexo estuarino de Paranaguá. De acordo com o IAPARDEs (2001) verifica-se ao norte deste estuá-
Tabela IV. Proporção sexual de $A$. brasiliensis em cada classe de comprimento.

\begin{tabular}{cccc}
\hline Classes de comprimento $(\mathrm{cm})$ & Fêmeas & Machos & $\chi^{2}$ \\
\hline $1,9-2,8$ & 0 & 0 & - \\
$2,9-3,8$ & 0 & 0 & - \\
$3,9-4,8$ & 0 & 0 & - \\
$4,9-5,8$ & 0 & 3 & 3,00 \\
$5,9-6,8$ & 2 & 5 & 1,29 \\
$6,9-7,8$ & 15 & 21 & 1,00 \\
$7,9-8,8$ & 32 & 41 & 1,11 \\
$8,9-9,8$ & 67 & 75 & 0,45 \\
$9,9-10,8$ & 103 & 98 & 0,12 \\
$10,9-11,8$ & 137 & 96 & $7,21 *$ \\
$11,8-12,8$ & 130 & 74 & $15,37^{*}$ \\
$12,9-13,8$ & 86 & 17 & $46,22^{*}$ \\
$13,9-14,8$ & 29 & 9 & $10,53^{*}$ \\
$14,9-15,8$ & 10 & 0 & $10,00^{*}$ \\
\hline
\end{tabular}

* Valores que representam diferenças significativas quando testados pelo método do $\chi^{2}(\alpha<0,05)$.

rio a coexistência de mangues bem desenvolvidos, atividades antrópicas como a presença de áreas desmatadas, pastagens e terras agricultáveis, além de baixa hidrodinâmica do local. Estas características, aliada ao alto índice pluviométrico e ao aporte de água doce da região (SUdERHSA 1998), favorecem a concentração de matéria orgânica ao norte do estuário, principalmente nos pontos amostrais localizados na baía de Guaraqueçaba (P1 e $\mathrm{P} 2)$, explicando os baixos valores de $\mathrm{pH}$ e, consequentemente, da transparência e do oxigênio dissolvido da região.

A influência do mar é um dos fatores que pode contribuir para o aumento do $\mathrm{pH}$ em ambientes estuarinos devido a característica alcalina dos oceanos $(\mathrm{pH}$ de aproximadamente 
8,2) (ODum 2004). Corroborando com esta prerrogativa observou-se maiores valores de $\mathrm{pH}$ e de salinidade nos pontos localizados ao sul do complexo estuarino de Paranaguá, região mais próxima ao mar aberto.

Foi verificado que não apenas as características específicas de cada ponto influenciaram o ambiente, mas também a oscilação da temperatura ao longo do período estudado. Assim, sugere-se que as variáveis ambientais, $\mathrm{pH}$, oxigênio dissolvido, transparência e salinidade estão mais relacionados às variações espaciais do ambiente, enquanto a temperatura com a variação temporal.

No complexo estuarino de Paranaguá, as maiores capturas foram obtidas no verão, corroborando os resultados registrados por Hostim-SıLva et al. (1995) na lagoa da Conceição, Santa Catarina, por Pessanha \& Araujo (2001) na baía de Sepetiba, Rio de Janeiro e por Neves et al. (2006) no manguezal de Guaratiba, Rio de Janeiro. Resultados diferentes foram encontrados por Bemvenuti (1987) na lagoa dos Patos, Rio Grande do Sul e por BARBIERI et al. (1991) na lagoa de Marapendi, Rio de Janeiro, os quais verificaram a maior captura no inverno e no outono, respectivamente. As diferenças verificadas nos trabalhos supracitados podem estar associadas às distintas metodologias empregadas na captura dos exemplares ou simplesmente porque cada estuário apresenta um padrão geomorfológico característico, o qual pode afetar os atributos físicos e químicos da água (Miranda et al. 2002) levando assim a uma resposta da biota de cada local. Além disso, os trabalhos foram realizados em anos diferentes aumentando a chance de eventos cíclicos, indetectáveis em estudos anuais, afetarem o número de indivíduos coletados.

No presente estudo, as grandes capturas ocorridas nas estações inverno (agosto) e outono (abril) no ponto P6 e no verão (janeiro) no ponto P7 foram consideradas atípicas, tratando-se possivelmente de captura acidental de cardumes. Em contrapartida, a baixa captura verificada no ponto P8 ocorreu, provavelmente, devido a uma menor tolerância da espécie às características próximas a do mar aberto, com alta hidrodinâmica e altos valores de salinidade. Embora a espécie em questão seja comumente registrada em áreas rasas da costa adjacente aos estuários, os registros de captura relatados por GodefroID et al. (1997) totalizou freqüência absoluta de 197 exemplares de $A$. brasiliensis, sendo considerado baixo o número de espécimes comparado com os obtidos no ponto P8 neste trabalho. Os autores supracitados obtiveram os exemplares, mensalmente, no período de um ano em uma praia adjacente ao complexo estuarino de Paranaguá, com petrecho de pesca semelhante ao utilizado neste estudo.

A amplitude do comprimento total determinado no presente trabalho para A. brasiliensis foi de $13,9 \mathrm{~cm}$, diferente dos $12,1 \mathrm{~cm}$ registrado por Hostim-Silva et al. (1995), na lagoa da Conceição, Santa Catarina, e dos $12,5 \mathrm{~cm}$ registrado por PESSANHA \& Araújo (2001), na baía de Sepetiba, Rio de Janeiro. Os pesquisadores supracitados na lagoa da Conceição utilizaram rede picaré (0,7 e $1,0 \mathrm{~cm}$ de malha), tarrafa $(1,0 \mathrm{~cm}$ de malha) e puçá $(0,5$ cm de malha), enquanto os pesquisadores na baía de Sepetiba realizaram capturas com rede picaré $(0,7 \mathrm{~cm}$ de malha). A diferença nos valores das amplitudes registrados para $A$. brasiliensis, estudadas em diferentes ambientes, pode não ser relevante e, provavelmente, ocorreu pela diferença dos tipos e das malhagens dos petrechos de pesca utilizados.

A análise em comprimento sazonal sugere a existência de um padrão cíclico de crescimento ao longo do ano, refletindo o período reprodutivo e de recrutamento de A. brasiliensis no sistema estudado. No verão observou-se a maior freqüência de indivíduos nas menores classes de comprimento, resultado do período reprodutivo ocorrido na primavera, quando se obteve os maiores indivíduos. A maior captura de indivíduos nas menores classes de comprimento (indivíduos jovens) foi também observada no verão por Bemvenuti (1987) e Hostim-Silva et al. (1995).

O predomínio de fêmeas nas maiores classes de comprimento sugere que as mesmas atingem maiores tamanhos que os machos. Esta diferença no comprimento foi relatada por BREDER \& Rosen (1966) como uma característica sexual secundária normal para os representantes da família Atherinopsidae.

A análise sazonal da massa corporal total obtida neste estudo responde pouco sobre a dinâmica da população no ambiente analisado. Entretanto, relacionando as análises sazonais de massa corporal média com a distribuição em classes de comprimento verifica-se no verão um predomínio de indivíduos de menor porte (formas jovens), indicando o recrutamento. Desta forma, mesmo sendo o verão o período de maior captura, a massa corporal média desta estação apresentou baixos valores. Estas análises conjuntas podem melhor explicar a dinâmica da população.

A análise do ciclo reprodutivo mostrou que o pico de atividade reprodutiva foi em outubro para as fêmeas e em setembro para os machos no complexo estuarino de Paranaguá. As fêmeas de $A$. brasiliensis tiveram o pico reprodutivo em novembro no Rio Grande do Sul (Bemvenuti 1987, Bervian \& Fontoura 1997), em setembro em Santa Catarina (Hostim-Silva et al. 1995), em outubro no Paraná (FÁvaro et al. 2003) e em julho no Rio de Janeiro (BARBIERE et al. 1991). Os resultados obtidos pelos autores supracitados sugerem que o período reprodutivo está relacionado com a temperatura, que de maneira geral aumenta quando a latitude diminui. Assim, há tendência deste evento ocorrer mais cedo durante o ciclo anual nas menores latitudes (Hostim-SiLvA 1994 in Bervian \& Fontoura 1997).

A análise dos resultados obtidos pelos pesquisadores anteriormente mencionados demonstra que, provavelmente, o desenvolvimento gonadal e o pico de atividade reprodutiva ocorram dentro da mesma faixa de temperatura nos diferentes locais estudados. Desta forma, sugere-se que estuários localizados em diferentes latitudes apresentem populações com diferentes padrões sazonais na estrutura em comprimento e em massa corporal.

O predomínio de fêmeas em relação a machos verificado no período reprodutivo (primavera) pode estar relacionado à

Revista Brasileira de Zoologia 24 (4): 1150-1156, dezembro 2007 
tática reprodutiva da espécie. Nossos dados corroboram com FAVARo et al. (2003) que encontraram a mesma proporção sexual durante o mesmo período.

No complexo estuarino de Paranaguá, o maior percentual de jovens no verão esteve associado ao processo reprodutivo da espécie, corroborado pela distribuição sazonal dos indivíduos em classes de comprimento, pela análise da massa corporal média sazonal e pela curva de maturação. Estes resultados corroboram com a freqüência percentual mensal de estádios de desenvolvimento ovariano e com a curva de maturação determinada para fêmeas e machos de A. brasiliensis na baía de Paranaguá (FAVARO et al. 2003).

Atherinella brasiliensis é uma espécie estuarina residente, mostrando-se amplamente distribuída nas áreas rasas estuarinas. $\mathrm{O}$ processo reprodutivo mostra-se intimamente relacionado com a temperatura e com as análises sazonais de estrutura em comprimento e em massa corporal. A referida espécie pode ser considerada chave em estudos e avaliações de impacto ambiental e/ou em ações de planos de manejo e conservação em ambientes estuarinos.

\section{REFERÊNCIAS BIBLIOGRÁFICAS}

Andreata, J.V.; A.G. Marca; C.L. Soares \& R.S. Santos. 1997. Distribuição mensal dos peixes mais representativos da Lagoa Rodrigo de Freitas, Rio de Janeiro, Brasil. Revista Brasileira de Zoologia 14 (1): 121-134.

Barbieri, L.R.R.; J.V. Andreata; M.A. Santos; M.H.C. Silva; A.S.C. Sebilia \& R.P. SANTOS. 1991. Distribuição e ciclo de vida das espécies de peixes mais abundantes da Laguna de Marapendi, Rio de Janeiro, Brasil. Revista Brasileira de Zoologia 7 (3): 223-243.

Bemvenuti, M.A. 1987. Abundância, distribuição e reprodução de peixes-rei (Atherinidae) na região estuarina da Lagoa dos Patos, RS, Brasil. Atlântica 9 (1): 5-32.

Bervian, G. \& N.F. Fontoura. 1997. Reprodução de Atherinella brasiliensis no Complexo Estuarino do Rio Tramandaí, Imbé, Rio Grande do Sul, Brasil (Teleostei, Atherinopsidae). Biociências 5 (2): 19-32.

Bigarella, J.J.; R.D. Becker; D.J. Matos \& A. Werner. 1978. A Serra do Mar e a porção oriental do Estado do Paraná - um problema de segurança ambiental e nacional (contribuição à geografia, geologia e ecologia regional). Curitiba, Governo do Estado do Paraná, Secretaria de Estado do Planejamento, Associação de Defesa e Educação Ambiental, 248p.

Breder, C.M. \& D.E. Rosen. 1966. Modes of reprodution in fishes. New York, Natural History Press, 941p.

FÁvaro, L.F.; S.C.G. Lopes \& H.L. Spach. 2003. Reprodução do peixe-rei, Atherinella brasiliensis (Quoy \& Gaimard) (Atheriniformes, Atherinidae), em uma planície de maré adjacente à gamboa do Baguaçu, Baía de Paranaguá, Paraná, Brasil. Re- vista Brasileira de Zoologia 20 (3): 501-506.

Figueiredo, J.L. \& N.A. Menezes. 2000. Manual de peixes marinhos do sudeste do Brasil. VI. Teleósteos (2). São Paulo, Museu de Zoologia, Universidade de São Paulo, 116p.

Godefroid, R.S.; M. Hofstaetter \& H.L. Spach. 1997. Structure of fish assemblage in the surf zone on the beach at Pontal do Sul, Paraná. Nerítica 11: 77-93.

Grasshoff, K; M. Ehrhardt \& K. Kremling. 1983. Methods of seawater Analysis. Weinhein, Verlar Chemie, $2^{\text {nd }}$ ed., 419p.

Hostim-Silva, M.; L. Clezar; G.C. Ribeiro \& C. Machado. 1995. Estrutura populacional de Xenomelaniris brasiliensis (Quoy \& Gaimard, 1824) (Osteichthyes - Atherinidae) na Lagoa da Conceição, SC, Brasil. Arquivos de Biologia e Tecnologia 38 (3): 949-960.

IAPARDES. 2001. Zoneamento da Área de Proteção Ambiental de Guaraqueçaba. Curitiba, Instituto Paranaense de Desenvolvimento Econômico e Social, 150p.

MAACK, R. 1981. Geografia física do Estado do Paraná. Rio de Janeiro, BDT/UFPR/IBPT, 350p.

Miranda, L.B.; B.M. Castro \& B. KJerfve. 2002. Princípios de oceanografia física de estuários. São Paulo, Universidade de São Paulo, 417p.

Neves, L.M; H.H. Pereira; M.R. Costa \& F.G. Araújo. 2006. Uso do manguezal de Guaratiba, Baía de Sepetiba, Rio de Janeiro, pelo peixe-rei Atherinella brasiliensis (Quoy \& Gaimard) (Atheriniformes, Atherinopsidae). Revista Brasileira de Zoologia 23 (2): 421-428.

Odum, E.P. 2004. Fundamentos de Ecologia. Lisboa, Editora Guanabara Koogan, $7^{a}$ ed., 927p.

Pessanha, A.L.M. \& F.G. Araújo. 2001. Recrutamento do peixerei, A. brasiliensis (Quoy \& Gaimard) (Atheriniformes, Atherinopsidae), na margem continental da baía de Sepetiba, Rio de Janeiro, Brasil. Revista Brasileira de Zoologia 18 (4): 1265-1274.

Santos, C.; R. Schwarz JR.; J.F. Oliveira Neto \& H.L. Spach. 2002. A Ictiofauna em duas Planícies de Maré do Setor Eurialino da Baía de Paranaguá, PR. Boletim do Instituto de Pesca 28 (1): 49-60.

Sokal, R.R. \& F.J. Rohlf. 1981. Biometry. San Francisco, Freeman, II+877p.

Spvs. 1992. Plano Integrado de Conservação para a região de Guaraqueçaba, Paraná, Brasil. Curitiba, Ícone Computação Gráfica, 129p.

Suderhsa. 1998. Atlas de recursos hídricos do estado do Paraná. Curitiba, Superintendência de Desenvolvimento de Recursos Hídricos e Saneamento Ambiental, 38p.

VAzzoler, A.E. DE M. 1996. Biologia da reprodução de peixes teleósteos: teoria e prática. Maringá, EDUEM, 169p.

Recebido em 19.VII.2007; aceito em 27.XI.2007. 